\title{
A Windowing-Recursive Approach for GPS Real-Time Kinematic Positioning
}

Zebo Zhou

Department of Surveying and Geo-informatics Engineering, Tongji University, 1239 Siping Road, 200092 Shanghai, P. R. China

E-mail: klinsmann.zhou@gmail.com

\section{Yunzhong Shen}

Department of Surveying and Geo-informatics Engineering, Tongji University, 200092 Shanghai, P. R. China Key Laboratory of Advanced Surveying Engineering of State Bureau of Surveying and Mapping, 1239 Siping Road, 200092 Shanghai, P. R. China

E-mail: yzshen@tongji.edu.cn

\section{Bofeng Li}

Department of Surveying and Geo-informatics Engineering, Tongji University, 1239 Siping Road, 200092 Shanghai, P. R. China

E-mail: Bofeng_Li@163.com

\begin{abstract}
All conventional Kalman filtering methods depend to a great extent on dynamic models for describing the motion state of vehicle. However, low cost GPS navigation systems do not provide velocity and acceleration measurements to construct dynamic models. Therefore, it is rather difficult to establish reasonable dynamic models. A windowing-recursive approach (WRA) which employs previous positions to predict the current position is proposed, the transition matrix is modeled for transforming the previous positions to the current one. Two typical transition matrices are constructed by numerical polynomial fitting and extrapolation. A real vehicular GPS experiment is carried out to demonstrate the WRA performances in two relative positioning scenarios. The data are processed by the least squares approach (LSA) and by WRA using the two developed transition matrices. The results show that the WRA performed excellently in a high sampling rate data. In case of a lower sampling rate, higher order polynomial fitting and extrapolation models work better than lower order models for a given window. In addition, the extrapolation models can alleviate the computation burdens significantly relative to the polynomial fitting models.
\end{abstract}

Key words: GPS; Transition matrix; Windowing-recursive approach; Kalman filter

\section{Introduction}

In GPS kinematic positioning at the meter-level accuracy, pseudoranges are preferred to phase measurements because they are free of cycle-slips. However, we still face many challenges for pseudorange utilization particularly in urban areas due to signal degradation, blockages, outliers and lack of measurements (Kuusniemi 
et al. 2004). The most important challenge in GPS navigation is how to efficiently improve the precision and reliability of real-time solution.

Integrated navigation systems, such as INS/GPS, GPS/DR (Dead Reckoning), are introduced mainly to overcome the limitation of each individual navigation sensor and improve the reliability (Chen and Cross 1990, Sameh et al. 2007, Umar et al. 2007, Logan et al. 2003). However, such integrations are too expensive to be extensively used in low-cost navigation campaigns. Another alternative, the so-called map matching technique has also been applied to assist positioning in areas without GPS signal reception (Mohammed et al. 2006). It requires a large number of updated map data and expensive hardware. The phase-smoothed code (Hatch 1982) was proposed to decrease the pseudorange noise by linearly combining phase and pseudorange measurements in the case of no cycle-slips. If cycle slips exist or the accuracy of phase-smoothed code is lower than that of Doppler measurements, Doppler-aided technique can be used further to smooth code and thus improve its accuracy (Cheng 1999). As an instantaneous measurement that directly relates to the relative movement between the receiver and satellite, Doppler shift has been widely used in velocity determination (Bruton et al. 1999, Zhang et al. 2006). Moreover, the position and velocity were trivially determined simultaneously by combining pseudorange and Doppler shifts (Mao et al. 2002).

In target tracking area, a time correlated acceleration function and its probability density variance were modeled by Singer (1970) in order to achieve optimal tracking performance, which was further developed by Zhou and Kumar (1984) by adding constraint condition of acceleration between adjacent epochs. The typical constant velocity and acceleration dynamic models and a "no dynamics" model were comprehensively discussed and compared for GPS/INS positioning based on vehicle trajectories (Schwarz et al. 1989). Recently, in the high-frequency motion conditions, discrete singular convolution algorithm has been proposed to determine the state equation (El-Diasty et al. 2006).

As an efficient sequential estimation algorithm, Kalman filter (KF) is extensively applied in navigation where observational errors and the predicted errors are assumed to be independent and normally distributed. Actually, unexpected errors exist in both state model and observation model. In order to balance the contributions of the state model and observation model to the final solution, some adaptively robust filters have been developed by Koch and Yang (1998) and Yang et al. (2001, 2002, 2003, 2005) in the frame of the robust M estimation (Huber 1981) and Bayesian estimation.

Since the low cost GPS navigation system cannot provide the velocity and acceleration measurements, it is rather difficult to establish a reasonable dynamic model. For this reason, we will develop an alternative windowing-recursive approach (WRA), where the current position vector is derived from several previous position vectors by using a transition matrix that does not rely on the velocity and acceleration. Section 2 will derive a general representation of WRA. Section 3 will construct two kinds of transition matrix by numerical polynomial fitting and extrapolation. Real vehicular GPS experiments are described in section 4 and the concluding remarks are given in section 5.

\section{General representation of windowing-recursive approach}

The trajectory of a moving object can reasonably be assumed to be smoothed in a short time span. Therefore, 
the position at the present epoch can be predicted more reliably with the positions of multiple historical epochs than with the position of only the latest epoch. Assuming that the time-window length contains $n$ epochs, we construct the transition equations as

$$
\boldsymbol{x}_{k}=\boldsymbol{\Phi}_{(k, k-n: k-1)} \boldsymbol{x}_{(k-n: k-1)}+\boldsymbol{w}_{k}
$$

where $\boldsymbol{x}_{k}$ denotes a state vector at epoch $k, \boldsymbol{x}_{(k-n: k-1)}$ is a column vector consisting of stacked state vectors from epoch $k$ - $n$ to epoch $k$ - 1 . The symbol $\boldsymbol{w}_{k}$ denotes a prediction error vector with the zero mean and covariance matrix $\Sigma_{w_{k}}$. The transition matrix at epoch $k$ is $\boldsymbol{\Phi}_{(k, k-n: k-1)}$, which transforms the states of the previous $n$ epochs into the current one.

Let $\Sigma_{\hat{\boldsymbol{x}}_{(k-n: k-1)}}$ be the covariance matrix of the historical state vector $\hat{\boldsymbol{x}}_{(k-n: k-1)}$, then the predicted position vector at epoch $k$ and its corresponding covariance are derived by

$$
\overline{\boldsymbol{x}}_{k}=\boldsymbol{\Phi}_{(k, k-n: k-1)} \hat{\boldsymbol{x}}_{(k-n: k-1)}
$$

and

$$
\Sigma_{\overline{\boldsymbol{x}}_{k}}=\boldsymbol{\Phi}_{(k, k-n: k-1)} \Sigma_{\hat{\boldsymbol{x}}_{(k-n: k-1)}} \boldsymbol{\Phi}_{(k, k-n: k-1)}^{T}+\Sigma_{\boldsymbol{w}_{k}}
$$

respectively. The linearized observation equations at epoch $k$ are symbolized as,

$$
\boldsymbol{l}_{k}=\boldsymbol{A}_{k} \boldsymbol{x}_{k}+\boldsymbol{e}_{k}
$$

where $\boldsymbol{A}_{k}$ is the design matrix of current state vector, and $\boldsymbol{l}_{k}$ is the measurement vector affected by normally distributed noise $\boldsymbol{e}_{k}$ with zero mean and co-variance matrix $\boldsymbol{R}_{k}=\sigma_{0}^{2} \boldsymbol{P}_{k}^{-1}$. The known prior variance scalar is $\sigma_{0}^{2}$ and $\boldsymbol{P}_{k}$ is the weight matrix of the measurements. By minimizing the weighted measurement residual vector $\boldsymbol{v}_{k}=\boldsymbol{A}_{k} \hat{\boldsymbol{x}}_{k}-\boldsymbol{l}_{k}$ and predicted residual vector $\overline{\boldsymbol{v}}_{\boldsymbol{x}_{k}}=\hat{\boldsymbol{x}}_{k}-\overline{\boldsymbol{x}}_{k}$, the Bayesian risk function is established by

$$
\min _{\boldsymbol{x}_{k}}: \boldsymbol{v}_{k}^{T} \boldsymbol{P}_{k} \boldsymbol{v}_{k}+\alpha_{k} \overline{\boldsymbol{v}}_{\overline{\boldsymbol{x}}_{k}}^{T} \boldsymbol{P}_{\overline{\boldsymbol{x}}_{k}} \overline{\boldsymbol{v}}_{\overline{\boldsymbol{x}}_{k}}
$$

where $\boldsymbol{P}_{\overline{\boldsymbol{x}}_{k}}=\sigma_{0}^{2} \Sigma_{\bar{x}_{k}}^{-1}$ denotes the weight matrix of predicted state vector. The symbol $\alpha_{k}$ is defined as an adaptive factor and generally we have $\alpha_{k} \neq 0$ (Yang and Gao 2005). The solution of minimal problem (2.5) is

$$
\begin{gathered}
\hat{\boldsymbol{x}}_{k}=\overline{\boldsymbol{x}}_{k}+\boldsymbol{K}_{k}\left(\boldsymbol{l}_{k}-\boldsymbol{A}_{k} \overline{\boldsymbol{x}}_{k}\right) \\
\Sigma_{\hat{\boldsymbol{x}}_{k}}=\frac{1}{\alpha_{k}}\left(\boldsymbol{I}-\boldsymbol{K}_{k} \boldsymbol{A}_{k}\right) \Sigma_{\overline{\boldsymbol{x}}_{k}} \\
\boldsymbol{K}_{k}=\frac{1}{\alpha_{k}} \Sigma_{\overline{\boldsymbol{x}}_{k}} \boldsymbol{A}_{k}^{T}\left(\frac{1}{\alpha_{k}} \boldsymbol{A}_{k} \Sigma_{\overline{\boldsymbol{x}}_{k}} \boldsymbol{A}_{k}^{T}+\boldsymbol{R}_{k}\right)^{-1}
\end{gathered}
$$

where $\Sigma_{\hat{\boldsymbol{x}}_{k}}$ is the covariance matrix of the Bayesian estimator. The correlation between $\hat{\boldsymbol{x}}_{k}$ and $\hat{\boldsymbol{x}}_{(k-n: k-1)}$ should be rigorously considered when the time window moves forward. Hereby, the covariance matrix $\Sigma_{\hat{\boldsymbol{x}}_{(k-n: k)}}$ is expressed as, 


$$
\Sigma_{\hat{\boldsymbol{x}}_{(k-n k)}}=\left[\begin{array}{cc}
\Sigma_{\hat{\boldsymbol{x}}_{(k-n k-1)}} & \Sigma_{\hat{\hat{k}}_{\hat{x}} \hat{x}_{(k-n k-1)}}^{T} \\
\sum_{\hat{\boldsymbol{x}}_{k} \hat{\boldsymbol{x}}_{(k-n k-1)}} & \Sigma_{\hat{\boldsymbol{x}}_{k}}
\end{array}\right]
$$

Rewriting (2.6) we obtain,

$$
\hat{\boldsymbol{x}}_{k}=\left(\boldsymbol{I}-\boldsymbol{K}_{k} \boldsymbol{A}_{k}\right) \overline{\boldsymbol{x}}_{k}+\boldsymbol{K}_{k} \boldsymbol{l}_{k}
$$

Replacing $\overline{\boldsymbol{x}}_{k}$ in (2.10) with (2.2) the alternative expression of (2.10) is

$$
\hat{\boldsymbol{x}}_{k}=\left(\boldsymbol{I}-\boldsymbol{K}_{k} \boldsymbol{A}_{k}\right) \boldsymbol{\Phi}_{(k, k-n: k-1)} \hat{\boldsymbol{x}}_{(k-n: k-1)}+\boldsymbol{K}_{k} \boldsymbol{l}_{k}
$$

Thus, the covariance matrix of $\hat{\boldsymbol{x}}_{k}$ and $\hat{\boldsymbol{x}}_{(k-n: k-1)}$ is derived from (2.11) by the law of error propagation as follows

$$
\Sigma_{\hat{\boldsymbol{x}}_{k} \hat{\boldsymbol{x}}_{(k-n: k-1)}}=\left(\boldsymbol{I}-\boldsymbol{K}_{k} \boldsymbol{A}_{k}\right) \boldsymbol{\Phi}_{(k, k-n: k-1)} \Sigma_{\hat{\boldsymbol{x}}_{(k-n k-1)}}
$$

It is rather easy to update the window vector $\hat{\boldsymbol{x}}_{(k-n+1: k)}$ and its corresponding covariance matrix $\Sigma_{\hat{\boldsymbol{x}}_{(k-n+1: k)}}$ which is a submatrix of (2.9). The WRA from epoch $k-1$ to $k$ is summarized with the following steps (see Fig.1):

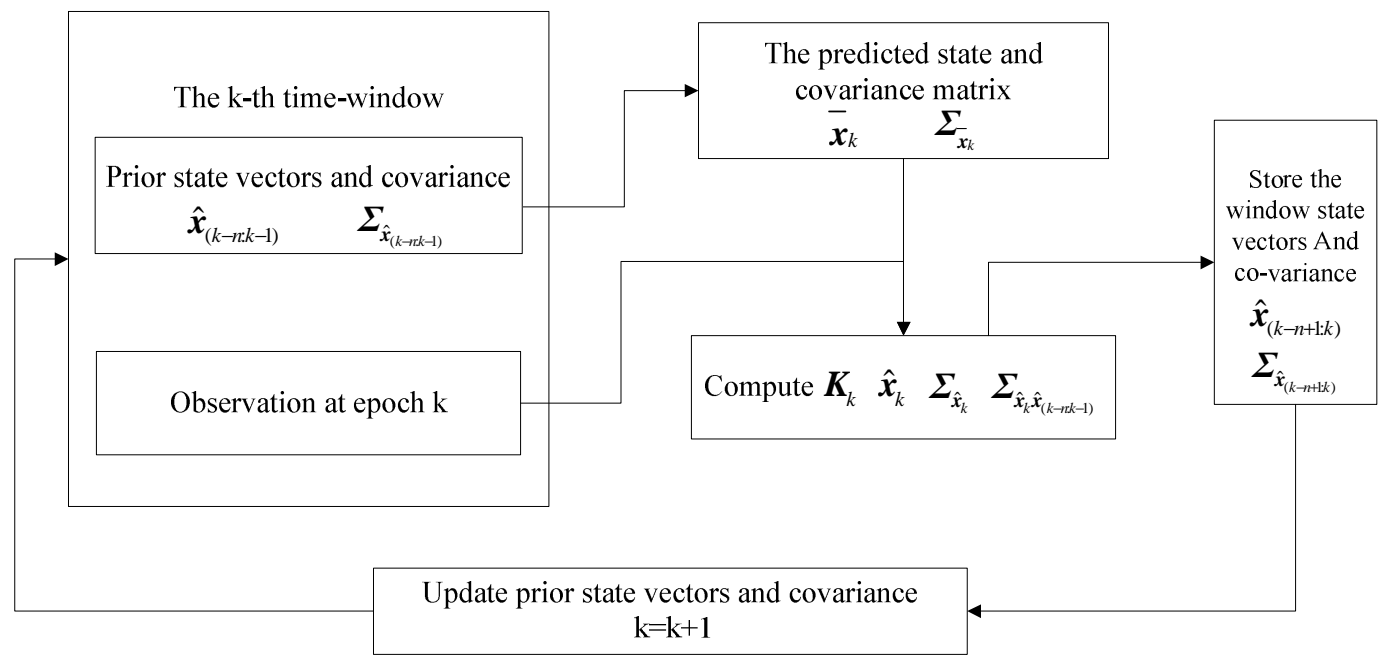

Fig. 1.The computation process of WRA

Step 1. Initialize the historical window information of $(n-1)$ epochs to achieve the position vector $\hat{\boldsymbol{x}}_{(k-n: k-1)}$ and its covariance matrix $\Sigma_{\hat{\boldsymbol{x}}_{(k-n k-1)}}$. Then compute the predicted results $\overline{\boldsymbol{x}}_{k}$ and $\boldsymbol{\Sigma}_{\overline{\boldsymbol{x}}_{k}}$ with (2.2) and (2.3);

Step 2. Compute $\boldsymbol{K}_{k}, \hat{\boldsymbol{x}}_{k}, \quad \Sigma_{\hat{\boldsymbol{x}}_{k}}$, and $\Sigma_{\hat{\boldsymbol{x}}_{k} \hat{\boldsymbol{x}}_{(k-n k-1)}}$ with (2.8), (2.6), (2.7) and (2.12) respectively;

Step 3. Update the window information, take the updated $\Sigma_{\hat{x}_{(k-n+1 k)}}$ out from (2.9) and store it along with the updated position vector $\hat{\boldsymbol{x}}_{(k-n+1: k)}$;

Step 4. If no interruption occurs, implement Step 1. Otherwise, re-initialize the window-recursive process from the epoch $k+1$. 
It is important to note that the position vectors of $n$ epochs in the initialization process are computed only by the GPS measurements based on the LS adjustment. The computation burden of WRA does not strongly depend on the window length, because the only inversion operation in (2.8) is not related to the window length. Additionally, if the dimension of state vector is smaller than that of the measurement vector, the equivalent recursive formulae can be constructed to decrease the computation burden similar to the equivalent $\mathrm{KF}$ formulae (Koch and Yang 1998). In the case of the window length $n=1$ the following properties are assigned to WRA:

(i) If $\alpha=1$, WRA is essentially equivalent to the classical KF.

(ii) If $\alpha=1$ and $\boldsymbol{P}$ is an equivalent weight matrix (Yang et al. 2001, Zhou 1989), WRA is equivalent to robust $\mathrm{KF}$.

(iii) If $\alpha=0$, WRA is equivalent to LS adjustment.

(iv) If $\alpha=0$ and $\boldsymbol{P}$ is an equivalent weight matrix, WRA is identical to robust LS adjustment.

\section{Modeling the transition matrix}

The most important benefit of WRA is using information of multiple historical epochs rather than only latest one. The different models are identified by the different transition matrices. In this section, we will establish two transition matrices by using the Newton interpolation model and polynomial fitting model.

\subsection{Extrapolation model}

The Newton interpolation and extrapolation method is a popular numerical analysis method, which is employed here to construct the transit matrix for its efficient performance. Assuming that the position vector is a function of time $t$, i.e., $\boldsymbol{x}=\boldsymbol{x}(t)$, and $\boldsymbol{x}_{i}$ denotes the position vector at epoch $t_{i}$ and $\delta t$ the time interval between consecutive epochs, the position at an arbitrary time can be expressed by the $n$-th order of Newton's forward differential extrapolation model as,

$$
\boldsymbol{x}\left(t_{k-n+s}, n\right)=\boldsymbol{x}\left(t_{k-n}+s \delta t, n\right)
$$

where $s$ denotes the $s$-th extrapolated epoch $(s \geq n)$ in time window and $n$ is the order of forward extrapolation. By expanding (3.1) and truncating the terms with order higher than $n$, we obtain

$$
\boldsymbol{x}\left(t_{k-n+s}, n\right)=\Delta^{1} \boldsymbol{F}_{0}+\frac{s}{1 !} \Delta^{2} \boldsymbol{F}_{0}+\frac{s(s-1)}{2 !} \Delta^{3} \boldsymbol{F}_{0}+\cdots+\frac{s(s-1) \cdots(s-n+2)}{n !} \Delta^{n} \boldsymbol{F}_{0}+\boldsymbol{R}_{n}(s)
$$

where $\boldsymbol{R}_{n}(s)$ is the high-order remainder term. Let $C_{s}^{n}=\frac{s !}{(s-n) ! n !}=\frac{s(s-1) \cdots(s-n+1)}{n !}$, then (3.2) 
can be simplified as

$$
\boldsymbol{x}\left(t_{k-n+s}, n\right)=C_{s}^{0} \Delta^{1} \boldsymbol{F}_{0}+C_{s}^{1} \Delta^{2} \boldsymbol{F}_{0}+C_{s}^{2} \Delta^{3} \boldsymbol{F}_{0}+\cdots+C_{s}^{n-1} \Delta^{n} \boldsymbol{F}_{0}
$$

where the small high-order term $\boldsymbol{R}_{n}(s)$ is disregarded. $\Delta^{m} \boldsymbol{F}_{0}$ with $m \in\{1,2, \ldots, n\}$ is derived from the following recursion formulae:

$$
\begin{aligned}
\Delta^{1} \boldsymbol{F}_{0}= & \boldsymbol{x}_{k-n} \\
\Delta^{2} \boldsymbol{F}_{0}= & \boldsymbol{x}_{k-n+1}-\boldsymbol{x}_{k-n} \\
\Delta^{3} \boldsymbol{F}_{0}= & \boldsymbol{x}_{k-n+2}-2 \boldsymbol{x}_{k-n+1}+\boldsymbol{x}_{k-n} \\
\Delta^{4} \boldsymbol{F}_{0}= & \boldsymbol{x}_{k-n+3}-3 \boldsymbol{x}_{k-n+2}+3 \boldsymbol{x}_{k-n+1}-\boldsymbol{x}_{k-n} \\
& \ldots \ldots \ldots \ldots \ldots \ldots \ldots \ldots \ldots \ldots \\
\Delta^{m} \boldsymbol{F}_{0}= & \sum_{i=0}^{m-1}(-1)^{m-1-i} C_{m-1}^{i} \boldsymbol{x}_{k-n+i}
\end{aligned}
$$

Specially, for $s=n$, equation (3.3) is rewritten as

$$
\boldsymbol{x}_{k}=\boldsymbol{x}\left(t_{k-n+s}, n\right)=C_{n}^{0} \Delta^{1} \boldsymbol{F}_{0}+C_{n}^{1} \Delta^{2} \boldsymbol{F}_{0}+C_{n}^{2} \Delta^{3} \boldsymbol{F}_{0}+\cdots+C_{n}^{n-1} \Delta^{n} \boldsymbol{F}_{0}
$$

Substituting (3.4) into (3.5), we obtain an alternative expression for $\boldsymbol{x}_{k}$ as

$$
\boldsymbol{x}_{k}=\sum_{i=0}^{n-1}\left\{C_{n}^{i} \sum_{j=0}^{i}\left[(-1)^{i-j} C_{i}^{j} \boldsymbol{x}_{k-n+j}\right]\right\}
$$

According to the equation $\sum_{i=1}^{n} a_{i}\left(\sum_{j=1}^{i} b_{j}\right)=\sum_{j=1}^{n} b_{j}\left(\sum_{i=j}^{n} a_{i}\right)$, we easily rewrite (3.6) to be

$$
\boldsymbol{x}_{k}=\sum_{j=0}^{n-1} \boldsymbol{J}_{j} \boldsymbol{x}_{k-n+j}
$$

where the coefficient $J_{j}$ reads

$$
J_{j}=\sum_{i=j}^{n-1}(-1)^{i-j} C_{i}^{j} C_{n}^{i} \quad j \in\{0,1, \ldots, n-1\}
$$

The matrix form of (3.7) is

$$
\boldsymbol{x}_{k}=\boldsymbol{J} \boldsymbol{x}_{(k-n: k-1)}
$$

where $\boldsymbol{x}_{(k-n: k-1)}=\left[\begin{array}{llll}\boldsymbol{x}_{(k-n)}^{T} & \boldsymbol{x}_{(k-n+1)}^{T} & \ldots & \boldsymbol{x}_{(k-1)}^{T}\end{array}\right]^{T}$ represents a $3 n$ column vector of positions for all $n$ epochs in the window, $\boldsymbol{J}=\left[\begin{array}{llll}J_{1} & J_{2} & \ldots & J_{n}\end{array}\right]^{T} \otimes \boldsymbol{E}_{3 \times 3}$ is a $3 \times 3 n$ constant coefficient matrix, and $\otimes$ is the Kronecker product. In real application, the matrix $\boldsymbol{J}$ is primarily computed offline and the coefficients for 
$2 \leq n \leq 5$ are presented in Table 1. Obviously, the transition matrix $\boldsymbol{\Phi}_{(k, k-n: k-1)}$ in the extrapolation model is specified by the matrix $\boldsymbol{J}$.

\subsection{Polynomial fitting model}

When the order of extrapolation is less than the number of epochs in the window, the elements of transition matrix should be estimated by LS. We use a polynomial fitting model here to construct the transition matrix.

Assuming the fitting order is $m(m<n)$, the polynomial model reads

$$
\begin{aligned}
& \boldsymbol{x}_{k-n}=\boldsymbol{a}_{1} \\
& \boldsymbol{x}_{k-n+1}=\boldsymbol{a}_{1}+\boldsymbol{a}_{2} \delta t+\boldsymbol{a}_{3} \delta t^{2}+\ldots+\boldsymbol{a}_{m} \delta t^{m-1} \\
& \ldots \ldots \\
& \boldsymbol{x}_{k-1}=\boldsymbol{a}_{1}+\boldsymbol{a}_{2}[(n-1) \delta t]+\boldsymbol{a}_{3}[(n-1) \delta t]^{2}+\ldots+\boldsymbol{a}_{m}[(n-1) \delta t]^{m-1}
\end{aligned}
$$

where $\boldsymbol{a}_{i}=\left[\begin{array}{lll}a_{i X} & a_{i Y} & a_{i Z}\end{array}\right]^{T}$ is the column vector to be estimated. Rewriting (3.10) in matrix form, one obtains

$$
\boldsymbol{x}_{(k-n: k-1)}=\boldsymbol{M a}+\boldsymbol{\varepsilon}
$$

where $\boldsymbol{M}=\left[\begin{array}{cccc}1 & 0 & \ldots & 0 \\ 1 & \delta t & \ldots & \delta t^{m-1} \\ \ldots & \ldots & \ldots & \ldots \\ 1 & (n-1) \delta t & \ldots & {[(n-1) \delta t]^{m-1}}\end{array}\right] \otimes \boldsymbol{E}_{3 \times 3}, \quad \boldsymbol{a}=\left[\begin{array}{c}\boldsymbol{a}_{1} \\ \boldsymbol{a}_{2} \\ \ldots \\ \boldsymbol{a}_{m}\end{array}\right]$, and $\boldsymbol{\varepsilon}$ is the normally distributed noise vector. Define the weight matrix as $\boldsymbol{P}_{\hat{\boldsymbol{x}}_{(k-n k-1)}}=\boldsymbol{\Sigma}_{\hat{\boldsymbol{x}}_{(k-n k-1)}^{-1}}$, and then the LS based optimal estimator $\boldsymbol{a}$ is calculated by

$$
\boldsymbol{a}=\left(\boldsymbol{M}^{T} \boldsymbol{P}_{\hat{\boldsymbol{x}}_{(k-n k-1)}} \boldsymbol{M}\right)^{-1} \boldsymbol{M}^{T} \boldsymbol{P}_{\hat{\boldsymbol{x}}_{(k-n k-1)}} \hat{\boldsymbol{x}}_{(k-n: k-1)}
$$

Denoting $\boldsymbol{u}=\left[\begin{array}{llll}1 & n \delta t & \ldots & (n \delta t)^{m-1}\end{array}\right] \otimes \boldsymbol{E}_{3 \times 3}$, the position of the current epoch is extrapolated using the fitted coefficients

$$
\boldsymbol{x}_{k}=\boldsymbol{u a}
$$

Substituting (3.12) into (3.13), we obtain

$$
\boldsymbol{x}_{k}=\boldsymbol{u}\left(\boldsymbol{M}^{T} \boldsymbol{P}_{\hat{x}_{(k-n k-1)}} \boldsymbol{M}\right)^{-1} \boldsymbol{M}^{T} \boldsymbol{P}_{\hat{\boldsymbol{x}}_{(k-n k-1)}} \hat{\boldsymbol{x}}_{(k-n: k-1)}
$$

Up to now, we have constructed the transition matrix $\boldsymbol{\Phi}_{(k, k-n: k-1)}=\boldsymbol{u}\left(\boldsymbol{M}^{T} \boldsymbol{P}_{\hat{\boldsymbol{x}}_{(k-n k-1)}} \boldsymbol{M}\right)^{-1} \boldsymbol{M}^{T} \boldsymbol{P}_{\hat{\boldsymbol{x}}_{(k-n k-1)}}$ for the 
polynomial fitting model. In principle, in the polynomial fitting model, the polynomial constraint is imposed on the positions of multiple epochs. Thus the polynomial fitting model is, to a certain extent, able to resist the outliers and thus decreases their influences on the final result in the time window. In addition, the polynomial fitting model is reduced to special cases in the following scenarios: (i) if $m=2$, the fitting model is equivalent to the constant velocity model; and (ii) if $m=3$ it is equivalent to the acceleration model. The polynomial fitting model is reduced to the Newton extrapolation model when no redundant point exists in the time window and $\boldsymbol{P}_{\hat{\boldsymbol{x}}_{(k-n k-1)}}$ is chosen as an identity matrix.

\section{Experiment and analysis}

The experiment data were collected with the sample interval of $1 \mathrm{~s}$ by two Topcon HiPer-Pro receivers on 22 April 2008. Although the longest distance between the reference and rover stations is less than $3 \mathrm{~km}$, various complex kinematic states are present. The double differenced observation model is used and the residual systematical errors are basically ignored due to their highly spatial correlation in such short distance. The C/A code measurements were utilized in the whole test. The trajectory is shown in Figure 2 and the velocities of the vehicle in the three components are in Figure 3. The precise results obtained by dual-frequency carrier phase are used as the actual values to evaluate the accuracies of the developed models.

In the following, we will investigate the accuracies of our WRA for different window lengths, fitting orders and sampling intervals. In all experiments the weight matrix $\boldsymbol{P}_{k}$ of double differenced GPS observations was rigorously derived from the identity weight matrix of undifferenced observations by using the law of error propagation. Different $\boldsymbol{w}_{k}$ assigned leads to different solutions, which means that the stochastic state model error should be reasonably estimated in real-time. Much research has been carried out to determine the stochastic model errors (Wang 2000, Moore and Wang 2003, Hewitson and Wang 2007, Li et al. 2008, Geng and Wang 2008). Yang and Gao (2005) also proposed an optimal algorithm to balance the contributions of state model and observation model. If better results are desired, some work related to quality control, e.g. outliers detection and isolation, model error estimation must be added and correctly done according to previous studies. It has been proved that these methods and algorithms are very effective and practical to achieve good and even optimal results. Therefore, without affecting the validity and efficiency of our WRA, we selected for simplicity the co-variance matrix $\Sigma_{w_{k}}$ as a $3 \times 3$ diagonal matrix with the element of $0.2 \mathrm{~m}^{2}$ according to some prior calculations. The extrapolation model and polynomial fitting model were used in the cases of $n=m$ and $n>m$, respectively.

The epoch-wise positions computed by LS approach are shown in Fig. 4(a) for the latter comparison purpose. 
Obviously, the worse LS solution is generally assigned to the larger PDOP value (see Fig. 5). The two constructed transition matrix models of WRA are examined in the window length $n$ from 2 to 5 with the fitting order $m$ from 2 to $n$, and the accuracies in three coordinate components are computed by

$$
\sigma_{X}=\sqrt{\frac{\sum_{i=1}^{q} \Delta X_{i}^{2}}{q}} \quad \sigma_{Y}=\sqrt{\frac{\sum_{i=1}^{q} \Delta Y_{i}^{2}}{q}} \quad \sigma_{Z}=\sqrt{\frac{\sum_{i=1}^{q} \Delta Z_{i}^{2}}{q}}
$$

where $\Delta X, \Delta Y, \Delta Z$ are the position differences between our WRA solutions and the actual ones, and $\sigma_{X}, \sigma_{Y}$ and $\sigma_{Z}$ are the accuracies. Here $q$ is the number of epochs. The computed accuracies are shown in upper part of Table 2. In order to demonstrate the performance of our WRA with contaminated data, satellite PRN 9 data was added with simulated outliers (15m) per 10 epochs. As shown in Fig. 4(b) the LS approach cannot resist the outliers and the accuracy of the solved positions dramatically falls down. The differences between the WRA solutions and actual values are illustrated in Fig. 6 and accuracies computed by (4.1) are presented in lower part of Table 2. It is observed from Table 2 that: (i) the WRA works as well as LS approach for processing clean raw data as long as the fitting order is higher than half of the window length; (ii) For processing outlier contaminated data, the WRA can obtain better results than LS approach. Especially in the case of the window length less than 4 epochs, the polynomial fitting model can usually get better results than the extrapolation model; (iii) The window length should be less than 5 epochs for the high sampling rate cases, since the longer window needs higher order fitting models which are easier to be affected by the outliers.

It is worth to point out that the developed extrapolation model is equivalent to the 'no dynamic' model that was elaborated in Schwarz (1989) in the case of $m=2$ and $n=2$. For this reason we do not compare our WRA with KF approach.

We further tested the performance of the WRA in the lower sampling rate case. The data is sampled by every 3 seconds and the test strategy is the same as the former experiments. The statistics are shown in Table 3. It is obvious that the accuracies of WRA in Table 3 are generally worse than those in Table 2 for low order models $(m \leq 3)$, but identical for high order models $(m \geq 4)$. This means that the high order polynomial fitting model or extrapolation model are more suitable in the low sampling rate cases if the outliers has been resisted by the robust algorithm.

\section{Concluding remarks}

In this paper, a general WRA has been proposed for GPS navigation. Two kinds of transition matrices are constructed to specify the extrapolation and polynomial fitting models. The real vehicular GPS experiments are implemented in cases of high and low sampling intervals. The results show that: (i) WRA with the fitting order higher than half of the window length works as well as LS approach in processing the data without any outlier, 
but WRA can achieve generally better results than LS approach in processing outlier contaminated data, especially in the case of the window length less than 4 epochs; (ii) in the high sampling case, the low order models can describe the motion states more adequately and achieve the optimal results in the window length less than 5 epochs. Conversely, in the low sampling case, the low order models cannot specify the motion states well enough and, thus high order models with longer window are necessary for achieving better performance. In other words, the time window length should be reasonably determined according to the kinematic state of different moving objects. In general, the varying velocity and acceleration of vehicle motion can be automatically specified in the high order fitting models of WRA and, therefore it does not need to construct the velocity and acceleration dynamical models for predicting the vehicular state epoch by epoch.

\section{Acknowledgements}

The work is partially sponsored by Natural Science Foundations of China (Grant No. 40674003, 40874016), and partially supported by the fund from the Key Laboratory of Advanced Surveying Engineering of SBSM (Grant No. TJES0809). The authors are very grateful to the anonymous reviewers for their constructive comments and suggestions.

\section{References}

Bruton A. M., Glennie C. L. and Schwarz K. P. (1999). Differentiation for high-precision GPS velocity and. acceleration determination. GPS Solutions, 2(4): 7-21.

Cheng P. (1999). Remarks on Doppler-aided smoothing of code ranges. Journal of Geodesy, 73: 23-28.

Chen W. and Cross P. A. (1990). Integration of GPS and an inertial system for precise surveying applications. Survey Review, 30(238): 375-395.

El-Diasty M., El-Rabbany A. and Pagiatakis S. (2006). New developments in state estimation for INS/GPS integrated systems. ION GNSS $19^{\text {th }}$ International Technical Meeting of the Satellite Division, Fort Worth, TX.

Geng Y and Wang J. (2008) Adaptive Estimation of Multiple Fading Factors in Kalman Filter for Navigation Applications. GPS Solutions, 12(4), 273-279.

Hatch R. (1982). The synergism of GPS code and carrier measurements. Proceedings of the third international geodetic symposium on satellite Doppler positioning, Las Cruces, NM, 2: 1213-1231.

Huber P. J. (1981). Robust statistics, John Wiley, New York.

Hewitson S. and Wang J. (2007) GNSS Receiver Autonomous Integrity Monitoring (RAIM) with a dynamic model, Journal of Navigation, 60(2), 247-263.

Koch K. R. and Yang Y. X. (1998). Robust Kalman Filter for Rank Deficient Observation Models. Journal of Geodesy, 72:436-441.

Kuusniemi H., Lachapelle G. and Takala J H. (2004). Positioning and velocity reliability testing in degraded 
GPS signal environments. GPS Solutions. 8:226-237, DOI 10.1007/s10291-004-0113-7.

Li B. F., Shen Y. Z. and Xu P. (2008). Assessment of stochastic models for GPS measurements with different types of receivers. Chinese Science Bulletin. 53(20):3219-3225.

Logan S. A., Leahy F. J. and Kealy A. (2003). Integration of GPS carrier phase and other measurements for kinematic mapping. Journal of Geodesy, 76: 543-556.

Mao X. C., Wada M. and Hashimoto H. (2002). Nonlinear filtering algorithms for GPS using pseudorange and Doppler shift measurements. The IEEE $5^{\text {th }}$ international conference on intelligent transportation systems, Singapore.

Mohammed A. Q., Robert B. N. and Washington Y. O. (2006). A high accuracy fuzzy logic based map matching algorithm for road transport. Journal of Intelligent Transportation Systems, 10(3): 103-115.

Moore M. and Wang J. (2003) An extended dynamic model for kinematic positioning. Journal of Navigation, 56(1), 79-88.

Schwarz K. P., Cannon M. E. and Wong R. V. C. (1989). A comparison of GPS kinematic models for determination of position and velocity along a trajectory. Manuscripta Geod, 14:345-353.

Singer R. A. (1970). Estimating optimal tracking filter performance for manned maneuvering targets, IEEE Transactions on Aerospace and Electronic Systems. 6(4): 473-483.

Umar I. B., Washington Y. O. and Shaojun F. (2007). Integrity of an integrated GPS/INS system in the presence of slowly growing errors. Part I: A critical review. GPS Solutions, 11:173-181.

Wang J. (2000) Stochastic modelling for RTK GPS/Glonass positioning, Navigation, Journal of the US Institute of Navigation, 46(4), 297-305.

Yang Y., He H. and Xu G. (2001). A new adaptively robust filtering for kinematic geodetic positioning. Journal of Geodesy, 75(2):109-116.

Yang Y. (2002). Robust estimator for correlated observations based on bifactor equivalent weights. Journal of Geodesy, 76: 353-358.

Yang Y. and Xu T. (2003). An adaptive Kalman Filter based on sage windowing weights and variance components. The Journal of Navigation, 56(2): 231-240.

Yang Y. and Gao W. (2005). Comparison of adaptive factors in Kalman Filter on navigation results. The Journal of Navigation, 58(3): 471-478.

Zhang J., Zhang K. F., Grenfell R. and Deakin R. (2006). Short note: On the relativistic Doppler effect for precise velocity determination using GPS. Journal of Geodesy, 80: 104-110.

Zhou H. R. and Kumar K. S. P. (1984). A current statistical model and adaptive algorithm for estimating maneuvering targets [J]. AIAA Journal of Guidance, 7(5): 596-602. 
Tables:

Table. 1 The coefficient $J$ for different window length ( $n=2$ to 5 )

\begin{tabular}{ccccc}
\hline Window length $n$ & & & \\
& $n=2$ & $n=3$ & $n=5$ \\
\hline$J_{1}$ & -1 & 1 & -1 & 1 \\
$J_{2}$ & 2 & -3 & 4 & -5 \\
$J_{3}$ & 3 & -6 & 10 \\
$J_{4}$ & & 4 & -10 \\
$J_{5}$ & & & 5 \\
\hline
\end{tabular}

Table. 2 Accuracies of all schemes for 1 second interval

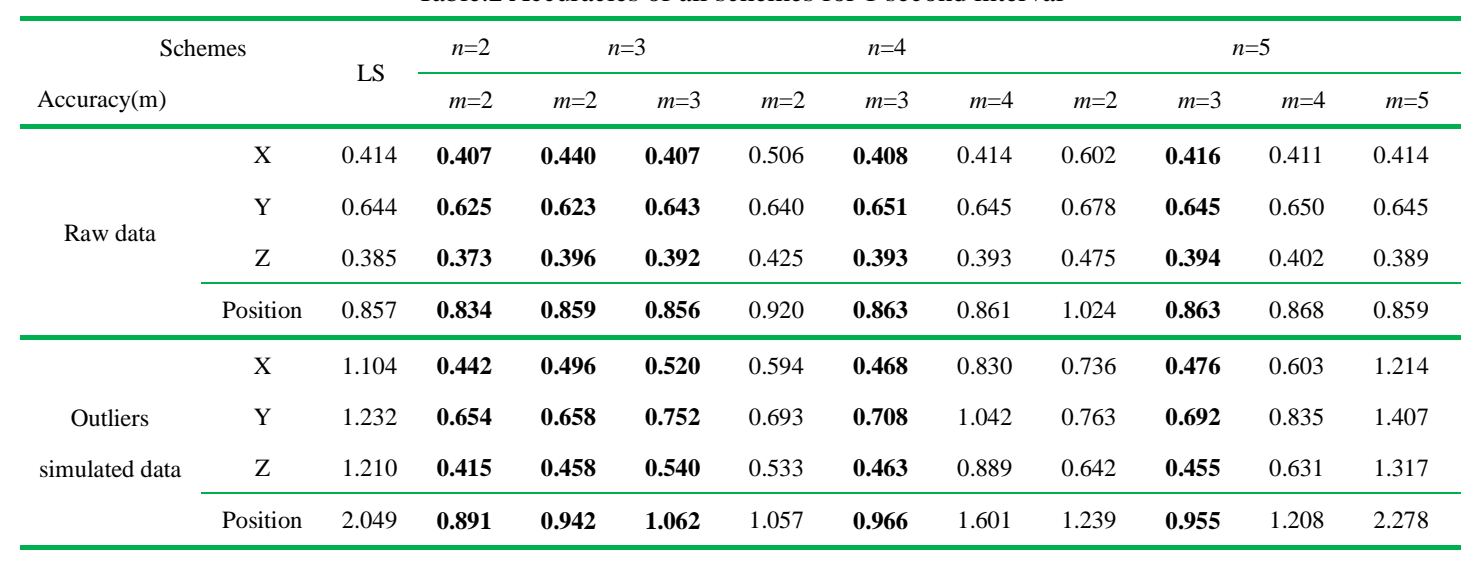

Table. 3 Accuracies of all schemes for 3 seconds interval

\begin{tabular}{|c|c|c|c|c|c|c|c|c|c|c|c|c|}
\hline \multirow{2}{*}{\multicolumn{2}{|c|}{$\begin{array}{l}\text { Schemes } \\
\operatorname{Accuracy}(\mathrm{m})\end{array}$}} & \multirow{2}{*}{ LS } & \multirow{2}{*}{$\begin{array}{l}n=2 \\
m=2\end{array}$} & \multicolumn{2}{|c|}{$n=3$} & \multicolumn{3}{|c|}{$n=4$} & \multicolumn{4}{|c|}{$n=5$} \\
\hline & & & & $m=2$ & $m=3$ & $m=2$ & $m=3$ & $m=4$ & $m=2$ & $m=3$ & $m=4$ & $m=5$ \\
\hline \multirow{4}{*}{ Raw data } & $X$ & 0.410 & 0.728 & 1.331 & 0.472 & 2.162 & 0.616 & 0.439 & 3.190 & 0.832 & 0.490 & 0.419 \\
\hline & $\mathrm{Y}$ & 0.665 & 0.768 & 1.152 & 0.661 & 1.776 & 0.763 & 0.652 & 2.548 & 0.847 & 0.662 & 0.663 \\
\hline & $\mathrm{Z}$ & 0.395 & 0.609 & 1.010 & 0.452 & 1.540 & 0.588 & 0.403 & 2.204 & 0.769 & 0.453 & 0.388 \\
\hline & Position & 0.876 & 1.220 & 2.029 & 0.930 & 3.194 & 1.143 & 0.883 & 4.640 & 1.414 & 0.940 & 0.875 \\
\hline \multirow{4}{*}{$\begin{array}{c}\text { Outliers } \\
\text { simulated data }\end{array}$} & $\mathrm{X}$ & 1.582 & 1.175 & 1.949 & 0.935 & 3.014 & 1.314 & 1.083 & 4.371 & 1.479 & 1.252 & 1.401 \\
\hline & $\mathrm{Y}$ & 1.703 & 1.057 & 1.595 & 1.028 & 2.344 & 1.123 & 1.341 & 3.28 & 1.292 & 1.308 & 1.628 \\
\hline & Z & 1.742 & 1.104 & 1.704 & 1.002 & 2.419 & 1.239 & 1.262 & 3.336 & 1.550 & 1.365 & 1.470 \\
\hline & Position & 2.905 & 1.928 & 3.040 & 1.713 & 4.520 & 2.126 & 2.136 & 6.406 & 2.501 & 2.268 & 2.603 \\
\hline
\end{tabular}


Figures:

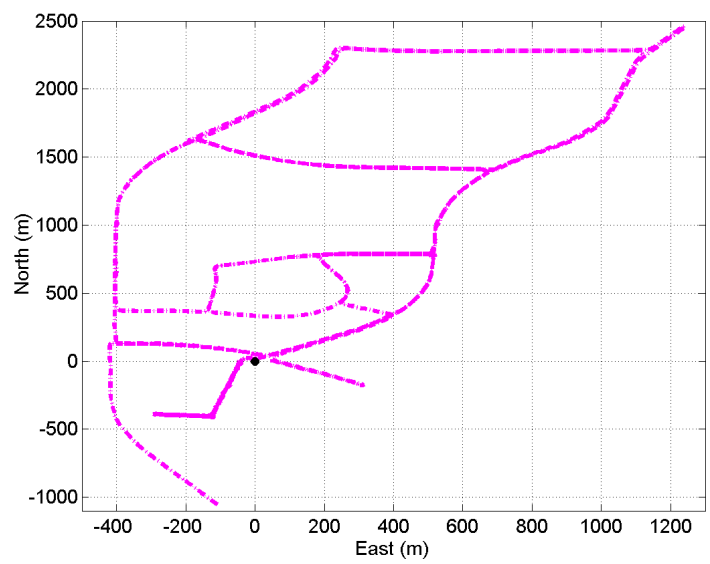

Figure 2. Vehicle trajectory
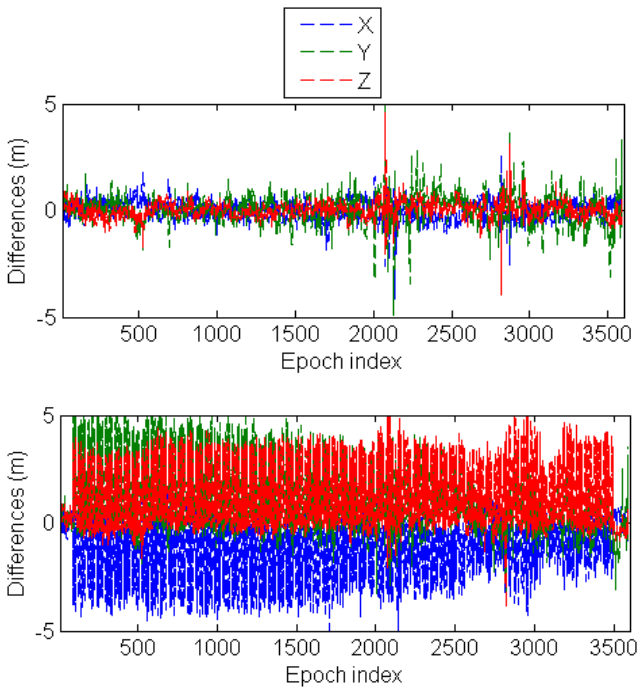

Figure 4a (top). Differences between LS solutions with raw data and the 'true' values

Figure $4 \mathrm{~b}$ (bottom). Differences between LS solutions with simulated outliers data and the 'true' values

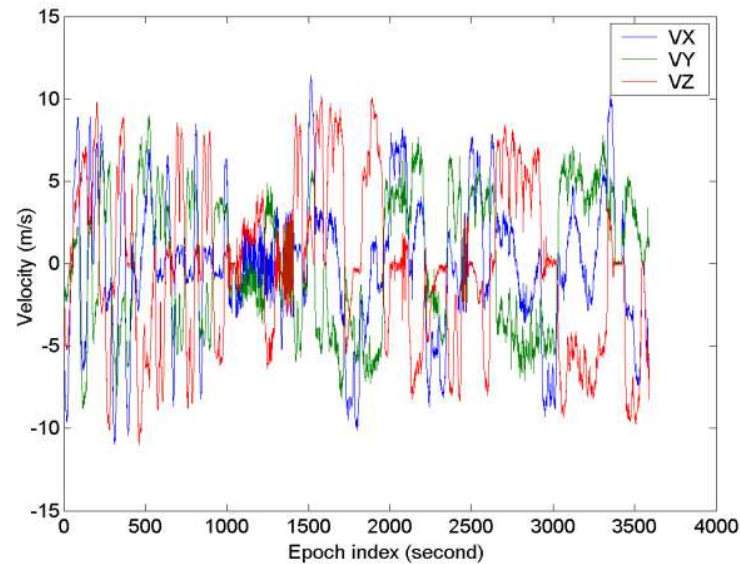

Figure 3. Velocities of X, Y, Z components

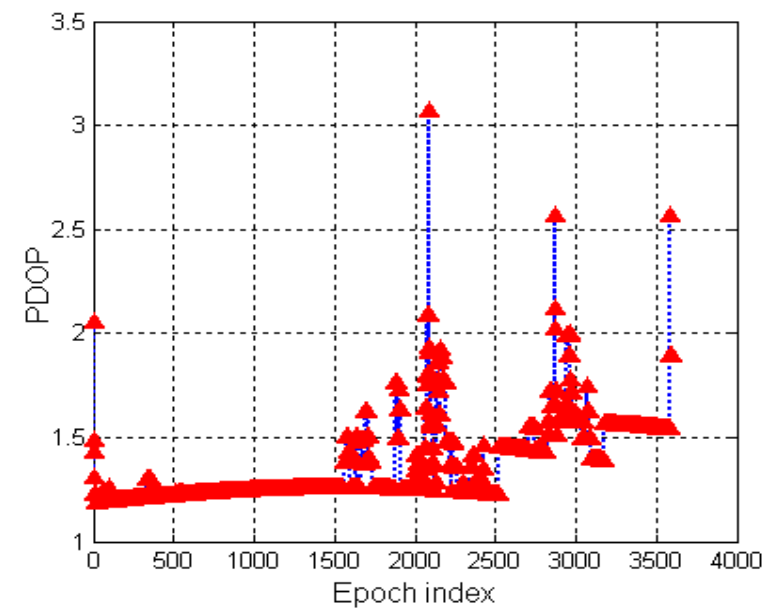

Figure 5. PDOP distribution for all epochs 


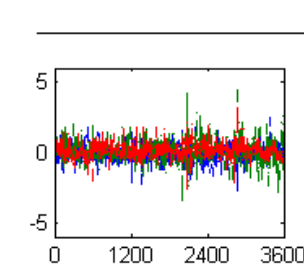

fitting order $m$ ranging from 2 to 5
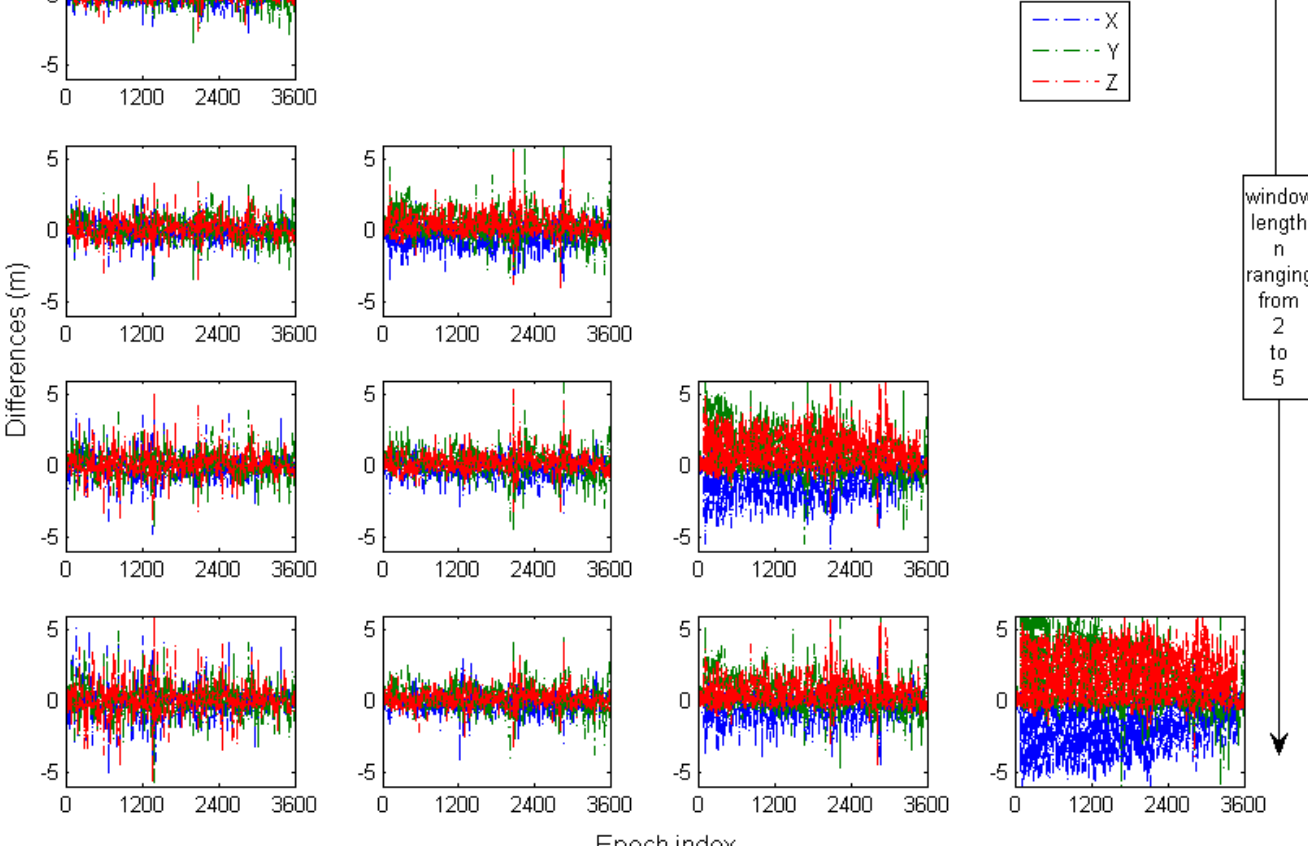

Figure 6. Differences between our WRA solutions and 'true' values for $1 \mathrm{~s}$ interval with outliers simulated data 